\title{
Winter maintenance-how to avert the emergency
}

\section{J. D. Kent}

This paper looks at two case studies where winter emergencies are averted by early closure of the road to traffic, and then considers whether these principles are relevant in a wider context. The paper questions whether the public attitude towards travelling in severe winter conditions is a major cause of disruption and discusses how this attitude can be changed.

\section{INTRODUCTION}

Normally when planning for emergencies it is not possible to make detailed plans for every possible event that could happen. As a result, emergency planning concentrates on dealing with the effects of an emergency. However, winter maintenance emergencies are unlike other emergencies, with the possible exception of flooding, in that you do get warning, you do have some idea of what is going to happen although not the exact location, and you can make appropriate preparations.

This is a double-edged sword. When flooding emergencies are forecast, at-risk locations can be evacuated and precautionary sand-bagging or construction of temporary dams undertaken. of snow, albeit that some disruption may be tolerated. If we are unable to keep traffic flowing and large numbers of vehicles become stranded on snow-covered roads with many motorists unable to complete their journeys, media coverage will report that 'councils have been caught out again'.

Traction control, all-wheel drive, heated windscreens, airconditioning-all isolate the driver from the effects of severe weather and promise greatly improved performance in snow compared to only a few years ago.

Similar advances have been made in snow-clearing equipment. Fifty years ago more severe winters were met with relatively basic methods and equipment (Figs 1 and 2). The limitations of these methods were obvious and road users accepted that there would be disruption in severe winter weather.

Today's equipment has hugely increased capability (Figs 3 and 4). Coupled with less severe winters this means that we are now far more able to tackle bad weather conditions but this ability does not come cheaply. The rising cost of snow-clearing
Apart from carrying out precautionary pregritting, no such large-scale activity can take place when a winter emergency is forecast. The roads authority can ensure that all plant is fully operational and that operatives are rested. The authority can possibly pre-arrange areas for tipping snow when the clearup eventually starts. But the major activity of snow clearing must wait until the snowfall actually starts.

Public expectation makes no allowance for this and in many ways public demands are ever-increasing. Flood prevention schemes can control and prevent flooding and in the same way we are expected to control the effects

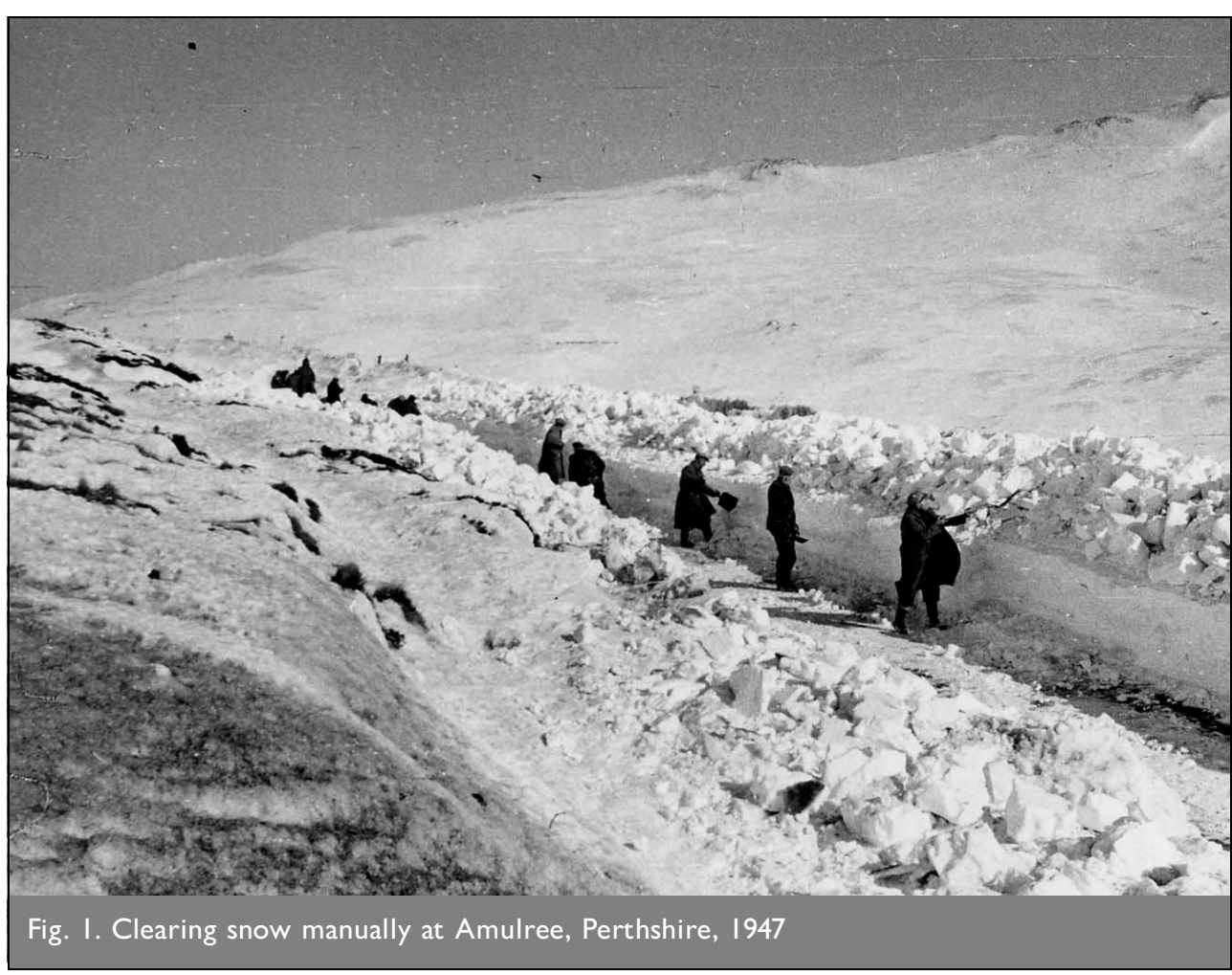




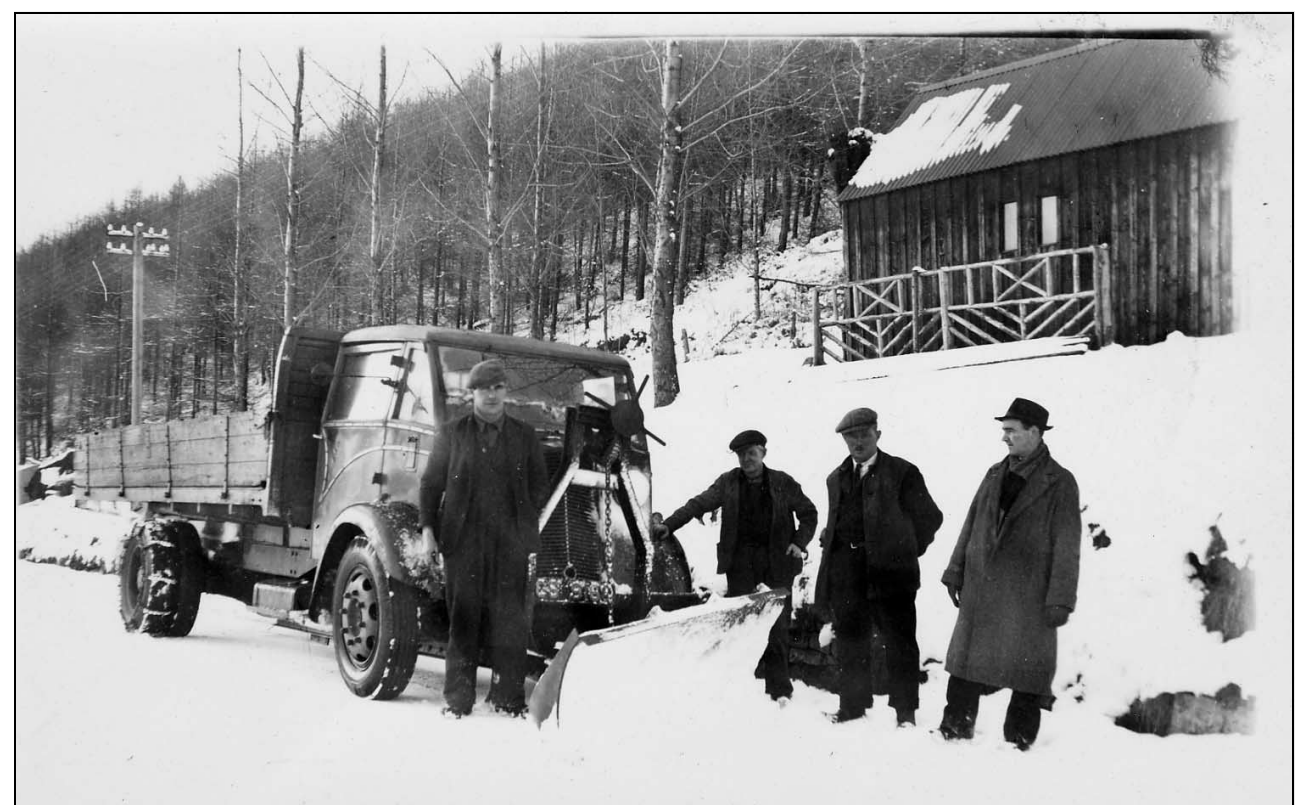

Fig. 2. Snowplough at Dalerb, Loch Tay, Perthshire, circa 1950
Faced with the option of providing additional plant which may be used only once or twice during a winter, it is not surprising that many authorities choose to operate a fleet level that cannot cope routinely with a severe snow event. They make a value judgement that allows them to deal with some but not all winter emergencies, with the consequence that closures and disruption are inevitable in the most severe conditions.

Warnings are issued to road users to make only essential journeys, and to take precautions such as carrying hot food and warm clothing. Little heed seems to be paid to this-the criterion appears equipment, due to the increases in technology and sophistication, together with pressure on budgets, results in roads authorities having to make difficult decisions about the level of resources that they can afford.
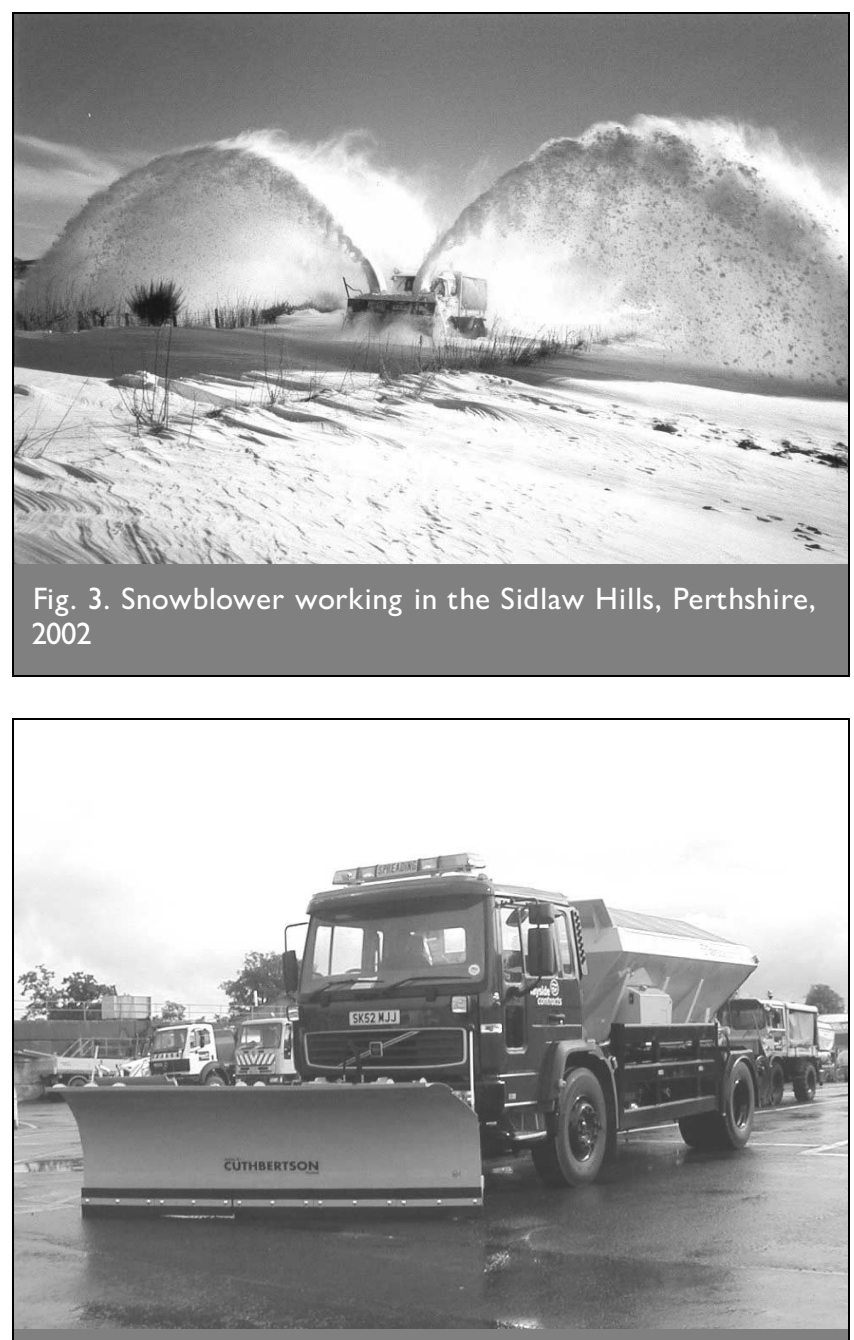

Fig. 4. Snowplough at Blairgowrie Depot, Perthshire, 2002 to be if a journey is 'desirable' rather than 'essential', and when drivers become stranded their major concern is usually more about when assistance will reach them than on the consequences of failing to complete their supposedly essential journey.

There is another difference between winter maintenance emergencies and other types of emergency. In most emergencies the people directly affected are mostly unwilling participants who happened to be in the wrong place at the wrong time. In winter maintenance, those caught up in the emergency are often there from their own choice, many already forewarned by a weather forecast. Even then, instead of cutting their journey short and going to a place of safety, they exacerbate the emergency by continuing until they can go no further and abandoning their vehicles, obstructing access for emergency or snow-clearing vehicles.

The following two case studies may be of interest. They both describe situations in the north of Perthshire in locations where the emergency can be predicted and appropriate precautions taken (Fig. 5). As a result the measures for dealing with the situation have been well-rehearsed and may give some useful suggestions on how to deal with other emergencies.

\section{CASE STUDY I-A93 GLENSHEE}

The Glenshee Ski Centre lies on the A93 Perth-Braemar road at an altitude of $660 \mathrm{~m}$. It lies just to the north of the boundary between two councils-Perth and Kinross Council and Aberdeenshire Council-and between two police forces-Tayside Police and Grampian Police. The majority of the traffic approaches from the south, on a winding road with steep gradients. The road from the north does not feature such steep gradients, although it is generally at high altitude.

This section of the A93 formerly included the infamous Devil's Elbow, which has now been bypassed. The realignment comprises a straight section with gradients varying between 

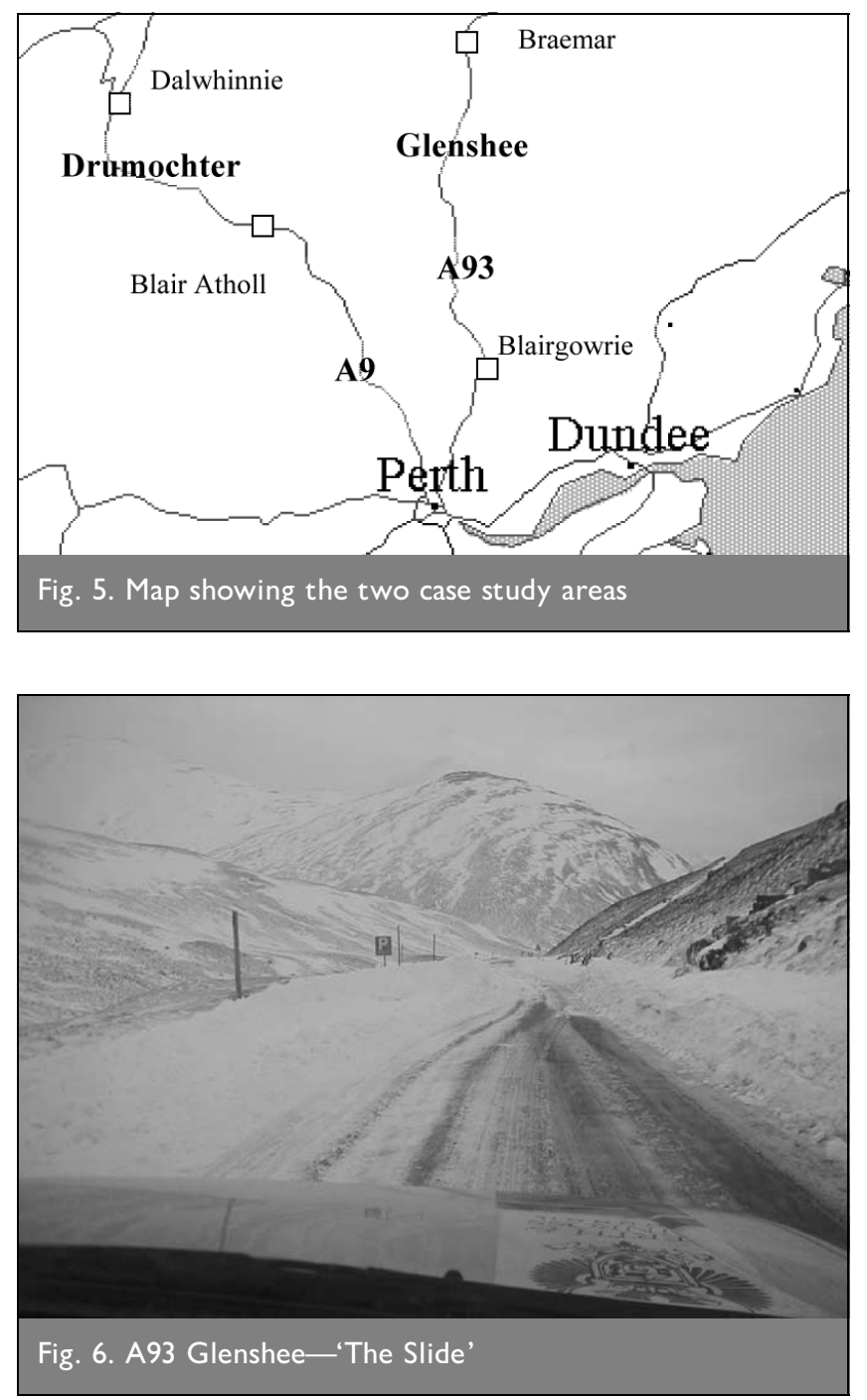

$10 \%$ and $12 \%$ over a length of $2 \mathrm{~km}$, known locally as 'The Slide' (Fig. 6).

In January 1984 heavy snow blocked the exit roads to north and south, trapping about 2000 skiers at the Ski Centre. They were able to shelter at the Centre buildings until the roads were reopened and they were evacuated, fortunately without loss of life.

Procedures were comprehensively revised to take account of the lessons learned and are described below.

\section{I. Mitigation}

Precautions taken to prevent a recurrence include stationing roads authority supervisors at the Centre when skiing is in progress and significant snowfall is anticipated. Close liaison takes place between the roads authorities, police and the Ski Centre operators.

\subsection{Road closure}

When conditions deteriorate to a level that causes concern, the slopes are closed and vehicles are convoyed out, led by a snowplough. The police then close snowgates to prevent access to the high-level section of road (Fig. 7).

A93 traffic flows are mainly during daytime with little overnight traffic, especially during the winter period. The hours

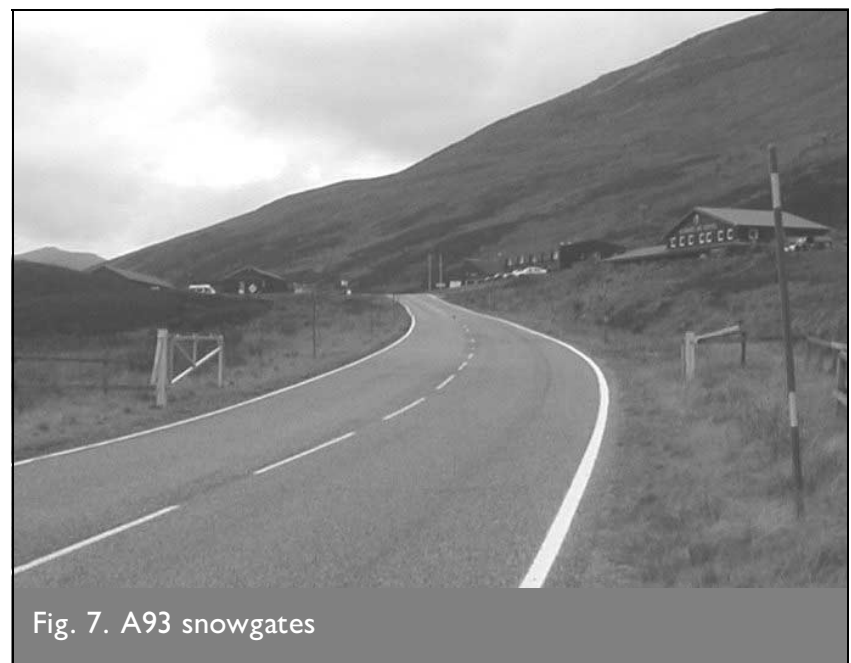

of cover for winter maintenance do not include overnight treatment and consequently the police will often close the snowgates as a precaution if conditions deteriorate during the hours of darkness.

\subsection{Reopening}

The road is normally reopened on the following morning by snowploughs in advance of the skiing traffic. If there is severe drifting, a snowblower is required which results in a delay to reopening the road to traffic. When the road is suitable for trafficking, the police open the snowgates.

\subsection{Monitoring and assessment}

Police, roads authorities and the Ski Centre operators hold a meeting each year to review the previous winter's experiences and decide on any changes which are necessary.

\section{CASE STUDY 2-A9 DRUMOCHTER SUMMIT}

In common with the A93 at Glenshee, the A9 at Drumochter is on the boundary between two councils - in this case Perth and Kinross Council and Highland Council—and two police forces, namely Tayside Police and Northern Constabulary. However, the A9 from Perth to Inverness is a trunk road and is the main arterial link to the north of Scotland.

Over the past 30 years the full length of 120 miles between Perth and Inverness has been realigned and improved, mainly to a standard of $7 \cdot 3 \mathrm{~m}$ single carriageway plus $0 \cdot 7 \mathrm{~m}$ edge strips, but with a number of dual carriageway sections.

Drumochter Summit is the highest point on the road at an altitude of $453 \mathrm{~m}$. It experiences severe winter weather which can render the road impassable. However, any closure to traffic has serious economic and social implications and so wellestablished measures have been developed to ensure that closures can be avoided as much as possible, and that when a closure is unavoidable the road can be reopened at the earliest opportunity.

\section{I. Mitigation}

Until recently, snowploughs patrolled continuously during the main winter period to ensure a prompt response if conditions deteriorated. The summit lay at the end of two adjacent gritting routes, partly a historic effect of the two council areas but also 


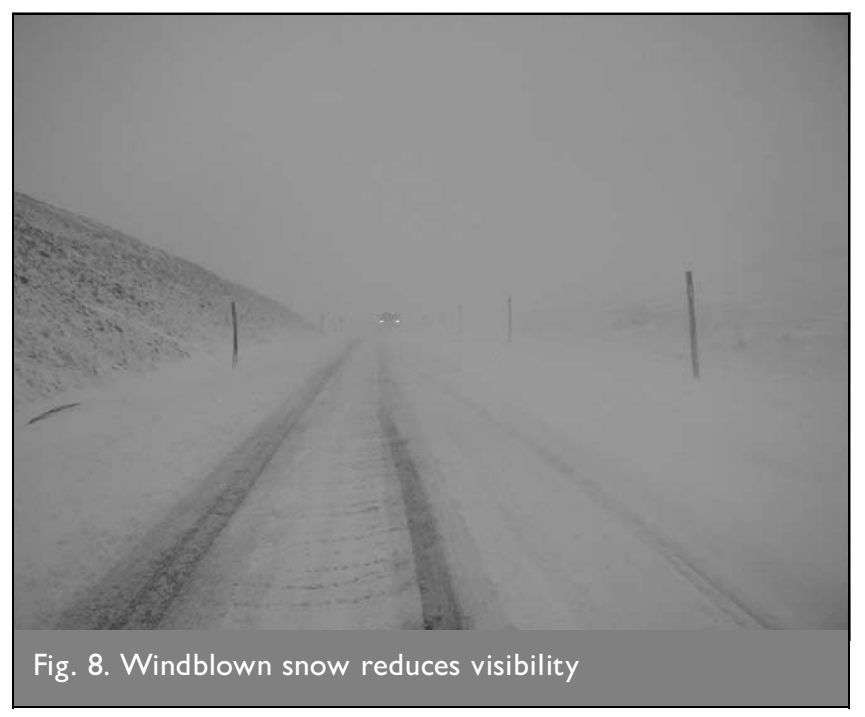

due to the location of depots and feasible route lengths. The weakness of this arrangement is a discontinuity in treatment at a point of severe weather. Scheduling patrol times so that the two ploughs would meet mitigated this.

Drumochter Summit is exposed to the south-west, which is also the direction of the prevailing wind. Much of the road is provided with a steel beam safety barrier which caused two problems: snow would settle in the sheltered lea of the barrier; and the barrier also trapped snow thrown clear by the snowplough blades. The combined effect was that the accumulation of snow could encroach onto the carriageway and on occasion it required the use of a snowblower to widen out the traffic lanes. The solution was to replace the beam barrier with a wire rope barrier which allowed snow to pass unhindered.

Snowgates have been erected to prevent traffic from entering the most severely affected section, together with folding 'Road closed' signs and warning lights. The National Driver Information Control Centre (NADICS) also gives out information on road conditions.

\subsection{Closure}

Problems usually start with severely restricted visibilitywhiteout conditions due to windblown snow and high winds (Fig. 8). Drivers may then drive into the snowdrifts at the side of the road or into oncoming traffic, or simply stop, leaving HGVs unable to restart on gradients or causing nose-to-tail pile-ups.

Police and snowplough drivers monitor conditions and when the police decide that the road should be closed, priority is given to preventing vehicles from entering the section.

The first vehicle (either roads authority or police) to arrive at each set of snowgates will hold traffic until police arrive. While police man the gates and turn traffic back, police and snowploughs check between the gates and assist any vehicles that are stranded. The gates are finally closed after all vehicles have been cleared.

The timing of the decision to close the gates is crucial. On some occasions when the decision has been taken too late (judged with the benefit of hindsight) as many as 200 vehicles have been stranded between the gates. It required several hours to dig out and escort these vehicles clear, with a knock-on effect on routine ploughing, and this delayed reopening the road.

\subsection{During a closure}

Snowploughing continues between the closed gates to keep the road clear for the eventual reopening. Even in the heaviest snow it is essential to keep a through-route clear. The snowplough drivers can achieve this with detailed local knowledge of the road and the absence of obstacles. In extreme conditions they may tackle dual carriageways in the wrong direction to get a downhill run at a snowdrift.

Regular meetings are held on site to assess conditions. The interval may be $3 \mathrm{~h}$ between meetings during a daytime closure. If the road is closed overnight a meeting at 6 am on the next day is normal.

There is considerable pressure to reopen the road at the earliest opportunity. The pressure comes from commercial interests, recreational (winter sports) traffic or social traffic.

The obvious question is 'When will the road be reopened?' It is impossible to answer this but it has been found useful to give out information on the NADICS variable message signs (VMS): 'Road closed-next update at 10 am'. Usually the time shown is 30-60 min after the next on-site meeting. Clearly it is essential to revise the VMS message regularly to retain credibility.

\subsection{Reopening the road}

When all parties decide that conditions are suitable for reopening, a time is decided-usually about $1 \mathrm{~h}$ later. Police organise the waiting vehicles into queues, often with HGVs at the front to break up hard-packed snow. Initially traffic is taken through in convoy with a snowplough at the front and police $4 \times 4$ at the rear. At this point it is actually an advantage that Drumochter Summit is at the end-point of two gritting routes, as this doubles up the number of ploughs available to help.

The decision to reopen is a subjective one. It involves assessing road and weather conditions at that time, together with an estimation of what the conditions will be like $1-2 \mathrm{~h}$ later when the traffic actually gets onto the road. There have been times when conditions have deteriorated during this interim period and it has been necessary to close again. The size of the convoy is a factor here. While convoys of about 150 vehicles have been run successfully, about 50-70 vehicles is better if the weather deteriorates.

\subsection{Monitoring}

All parties meet prior to the start of each winter. The closures during the previous winter are discussed and reviewed (a detailed log is kept of each closure) and the procedure changed where necessary.

After so many years of successful operation, any changes are relatively minor and often reflect changing circumstances. For example, the relocation of one set of snowgates is being considered to take advantage of a recently constructed car park at a tourist attraction. This may be suitable for parking HGVs and relieve congestion in the adjacent village of Blair Atholl. 
Another possible improvement is the preparation of a leaflet for issue to stranded drivers to explain the reasons for convoying, how to drive in a convoy and what to do if conditions deteriorate.

Perhaps the biggest change is that personnel from Highland Council and Perth and Kinross Council are no longer involved. Since April 2001 the Scottish Executive has employed BEAR (Scotland) Ltd to provide the maintenance service on trunk roads in the northern part of Scotland. This has removed a significant base of experience and BEAR is on a sharp learning curve. In general the procedures used are similar to those described here, although communications should be more straightforward in that a single organisation (i.e. BEAR) has replaced two councils.

\section{CONCLUSIONS}

\section{I. So what lessons can be learned from these two case studies?}

The road closures at these locations are well-rehearsed to the extent that they should possibly not be considered emergencies. Perhaps the real emergency would only occur if the road were not closed in time. Anticipating that conditions are deteriorating, and then at a critical point stopping vehicles from going into these areas, averts the emergencies.

These roads have several features which facilitate this method of operation.

(a) The locations of the worst points-that is, the points of closure - can be identified in advance.

(b) Traffic can be held in safe locations fairly close to the point of closure.

(c) The roads are essentially linear routes with few points of access along the route.

(d) These locations have a reputation, almost a notoriety, and there is a recognition that the roads cannot be kept open at all times.

(e) The roads are closed as a precautionary measure to allow the earliest possible reopening when conditions improve.

Most of these features are not present when widespread heavy snowfall affects a wide area.

(a) There is no specific point of closure, or if there is, it is often not possible to identify it in advance.

(b) There are no convenient points to stop traffic and turn them back.

(c) There are no convenient safe areas to hold vehicles waiting for the road to reopen.

(d) There is no easy way of physically controlling the closure of the road.

(e) The roads become closed by virtue of stranded vehicles which obstruct passage.

This places a demand on the emergency services and civil authorities to care for stranded motorists and remove stranded vehicles, and obstructs snow clearing with a consequent delay in reopening roads.

Perhaps the only way of applying the principles of the Glenshee and Drumochter road closures to the wider situation is to remove the traffic by voluntary means - that is, by persuading drivers not to travel in the first place.

Interestingly, a colleague of the author recently took issue with a national newspaper which criticised snow-clearing efforts and praised the bravery of motorists who ventured out not knowing if they would be able to complete their journeys. His letter was published, but with the deletion of a paragraph which pointed out how ineffective a snowplough was when caught in stranded traffic and suggested that matters would only improve when drivers accepted that they themselves were a major cause of the problem. Clearly a bridge too far.

Even when people have suitable clothing, they often seem reluctant to actually wear it, viz. skiers from Glenshee who encounter snow problems on the road. Instead of donning their skiing clothing, they venture out of their stranded cars wearing only light clothing, totally unsuited to the conditions.

\subsection{Public information}

It could be argued that improving the information given to the public would result in motorists avoiding the area or simply not travelling at all in poor weather conditions. However, this is only part of the picture.

While accurate, up-to-date and, above all, reliable information is essential, a major shift in public attitude is also needed. If we are encouraging road users not to travel when the forecast is poor, employers need to accept that employees may be late or not come in at all. Too often the attitude is 'I made it, so why couldn't you?'

Many businesses operate on just-in-time deliveries for greater efficiency. Even if they were able to reschedule deliveries in advance of a period of poor weather, they would not be able to cope with the extra stock. It is essential that deliveries get through on time and rather than abort a delivery on the basis of a report of poor weather or road conditions, they will continue with their activities until physically prevented from doing so.

Many drivers will ignore warning signs or radio broadcasts saying that a road is closed: they don't believe it and want to see for themselves. Often other motorists will follow, thinking that the lead driver knows an alternative route. And sometimes they find out that the road isn't closed after all. We must not underestimate the importance of this. We must make sure that all of the information put in the public domain is accurate and find some way of controlling 'rogue' broadcasts which bring public information into disrepute.

The growth in mobile phone usage has provided an unofficial road situation reporting network for radio stations which supplements official reporting methods. However, the scale of mobile phone reporting is also its weakness. Reports are unregulated and lack balance or priority. They are unchecked and not made to any set criteria.

We need to develop a system which provides more comprehensive, more accurate, more up-to-date information. We need 
to be more proactive and feed this information to the radio stations to reduce their reliance on the 'volunteer' information.

We must change public perception. In the same way that drinkdriving is now socially unacceptable, and that the attitude to speeding in built-up areas is changing, we have to change the attitude to driving in bad weather conditions. Drivers who disregard snow warnings and then have to be rescued should not be regarded as heroes.

The difficulties of changing public perception in this way are considerable. The drink-driving campaign was sustained over many years to achieve the current attitude. A recent campaign by the Scottish Executive to persuade drivers to use healthier alternatives to driving- -you can do it-you can let go of the steering wheel'-was criticised in the media as an admission that the Executive has not succeeded in resolving transport problems. It would appear that payment of the motoringrelated taxes gives each and every driver the right to drive wherever and whenever he or she wants, regardless of the consequences.

There is a further difficulty of changing the perception to snow emergencies. Severe snow conditions occur on relatively few occasions each winter and even then are rarely countrywide. A campaign will only be relevant if it is timed to match these conditions.

With this background it is difficult to see how an effective sustained campaign could be mounted, or indeed if there is political will to fund and carry through such a campaign. But despite these difficulties we cannot afford not to delay the process.
The trend of technological improvement will continue and indeed the pace will accelerate. Fifteen years ago central door locking systems were only to be found on a limited number of cars at the upper end of the market. Now central locking is commonplace on all but the most basic models and in the same way we can expect to find traction control and anti-lock braking systems as standard fittings in the future.

We can also expect to see wider use of in-car GPS route-finder systems which direct the driver to his or her destination. Can we feed information on road conditions into this process, to influence the route-finder program? Can account be taken of a route's susceptibility to severe weather, or of the level of service provided? Is it possible to include weather information, either forecast or real time, or information on actual treatment, transmitted from transponders fitted to winter maintenance vehicles?

There will be similar advances in the sophistication and performance of winter maintenance plant and the combined effect of these developments will be to provide road users with the desired improvements in driving conditions - in most circumstances. But as much as we can mitigate the effects of winter weather, it would be unrealistic to expect that we will be able to eliminate them completely. Disruption will still occur in the most severe weather and it is vital that we start the process to change drivers' perceptions.

We need to work with the media on this. We need to show them what we are doing and, more importantly, what we are not able to do. We also need to accept that we are not media professionals, and learn from other people who have succeeded in changing public opinion.

Please email, fax or post your discussion contributions to the secretary by I June 2003: email: kathleen.hollow@ice.org.uk; fax: +44 (0)20 7799 I325; or post to Kathleen Hollow, Journals Department, Institution of Civil Engineers, I-7 Great George Street, London SWIP 3AA. 